\title{
Numerical Simulation of Driven Convective Heat Transfer Based Lattice Boltzmann Method in a Porous Cavity
}

\author{
You-Sheng Xu, ${ }^{1}$ Rui-Min Wang, ${ }^{2}$ Guo-Neng Li, ${ }^{1}$ and You-Qu Zheng ${ }^{1}$ \\ ${ }^{1}$ School of Mechanical \& Automotive Engineering, Zhejiang University of Science and Technology, Hangzhou 310023, China \\ ${ }^{2}$ Department of Science and Technology, Jinhua Polytechnic, Jinhua 321017, China \\ Correspondence should be addressed to You-Sheng Xu; 113003@zust.edu.cn
}

Received 5 September 2014; Accepted 23 March 2015

Academic Editor: Charles M. Drain

Copyright (c) 2015 You-Sheng Xu et al. This is an open access article distributed under the Creative Commons Attribution License, which permits unrestricted use, distribution, and reproduction in any medium, provided the original work is properly cited.

\begin{abstract}
A lattice Boltzmann model of the uniform velocity, driven convective thermal conductivity in a porous cavity is studied. The Darcy, Richardson, and Reynolds numbers are shown to have a significant influence on the heat transfer behavior and the horizontal velocity of the flow field, while the porosity has little influence on either. The model is validated by the average Nusselt number at different Reynolds numbers, and the numerical results are in good agreement with available published data.
\end{abstract}

\section{Introduction}

Driven convective heat transfer in porous cavities has become a topic of interest in textile fiber and engineering fields $[1,2]$ through efforts to improve chemical and petroleum engineering, cooling of electronic devices [3], food processing, and functional clothing design.

Over the past several decades, convection heat transfer in porous media has been studied both experimentally and theoretically, but there have been relatively few numerical investigations. A coupled lattice Bhatnagar-Gross-Krook (CLBGK) model using the Boussinesq equations to simulate fluid flow in porous media was proposed by Guo et al. [4] and by other researchers. Guo and Zhao used the representative elementary volume (REV) scale [5] to study driven convection at a uniform velocity. The REV scale is much larger than the pore scale but much smaller than the domain scale. A number of other models have also been proposed, such as the Darcy model, the Brinkman-extended Darcy model, and the Forchheimer-extended Darcy model [5]. These differ from conventional numerical methods such as the finite-difference, finite-volume, and finite-element methods. Recently, a generalized lattice Boltzmann equation (GLBE) was proposed for isothermal incompressible flows in porous media [5].
The aim of the present paper is to study the effects of Darcy number $(\mathrm{Da})$, porosity $(\varepsilon)$, Reynolds number (Re), and Richardson number (Ri) on the driven convection using GLBE-based simulations. We adopt a model in which the sidewalls of the porous cavity are at different temperatures. To the best of our knowledge, previous simulations of driven flow have been limited to isothermal problems solved using lattice Boltzmann methods (LBMs); there have been no reports of studies using an LBE model. The flow and heat transfer in porous media are coupled by rather complicated mechanisms by extending the GLBE [5] proposed to thermal flows. We use the GLBM to simulate the velocity field and use a newly defined temperature distribution function LBM to simulate the temperature field.

We discuss the physical problem and mathematical formulation of porous flow in Section 2, and, in Section 3, the proposed LBM formulation including the proposed treatments of the velocity and temperature boundary conditions is presented. The numerical results and a conclusion are given in Sections 4 and 5, respectively.

\section{Mathematical Formulation}

Over the past decades, the lattice Boltzmann method (LBM) has been developed considerably for simulating fluid flows 


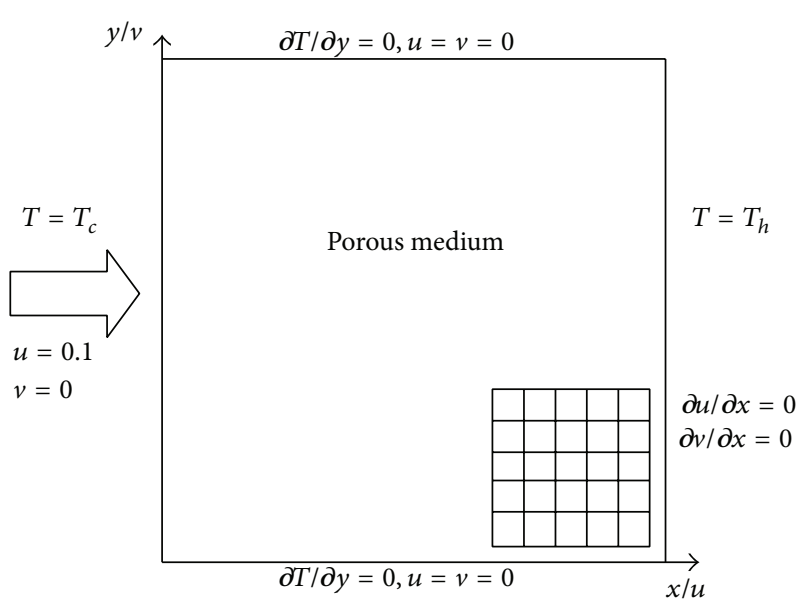

FIGURE 1: Schematic of the physical model and coordinate for the problem with boundary conditions.

and heat transfer phenomena in porous media $[5,8-16]$. The physical problem is illustrated schematically in Figure 1.

A square cavity is filled with an isotropic, homogeneous porous medium that is heated by the two vertical sidewalls. The fluid is assumed to flow into the porous cavity from the left-hand side. The (dimensionless) temperatures of the hot and cold sidewalls are $T_{h}=0.5$ and $T_{c}=-0.5$, respectively. The medium is also assumed to have a constant porosity. The governing equation of the generalized model for the incompressible fluid flow and heat transfer in such a porous media is given as

$$
\begin{aligned}
\nabla \cdot u & =0 \\
\frac{\partial u}{\partial t}+u \cdot \nabla\left(\frac{u}{\varepsilon}\right) & =-\nabla(\varepsilon p)+\frac{1}{\operatorname{Re}_{e}} \nabla^{2} u+F \\
\sigma \frac{\partial T}{\partial t}+u \cdot \nabla T & =\frac{1}{\operatorname{PrRe}} \nabla^{2} T,
\end{aligned}
$$

where $u$ and $p$ are the volume-averaged velocity and pressure, respectively. $\varepsilon$ is the porosity of the medium. Consider $\sigma=$ $\varepsilon+(1-\varepsilon) \rho_{s} c_{p s} / \rho_{f} c_{p f}$ represents the ratio between the heat capacities of the solid and fluid phases with $\rho_{s}\left(\rho_{f}\right)$ and $c_{p s}\left(c_{p f}\right)$ being the density and capacity of the solid (fluid) phase, respectively. In this work, the Reynolds number $(\operatorname{Re}=L U / v)$ is assumed to be equal to the effective Reynolds number $\mathrm{Re}_{e}$. The Prandtl number is Pr. The last term $(F)$ on the righthand side of (1b) represents the total body force due to the presence of a porous medium and other external force fields and is expressed as

$$
F=-\frac{\varepsilon}{\mathrm{DaRe}} u-\frac{\varepsilon F_{\varepsilon}}{\sqrt{\mathrm{Da}}} P u \mid u+\frac{\varepsilon \mathrm{Gr}}{\operatorname{Re}_{e}^{2}} k T
$$

where $\mathrm{Da}$ is Darcy number defined as $\mathrm{Da}=K / L^{2}$, Gr is the Grashof number, $k$ is the unit vector in the $y$-direction, and $F_{\varepsilon}$ is a geometric function defined as [17]

$$
F_{\varepsilon}=\frac{1.75}{\sqrt{150 \varepsilon^{3}}} \text {. }
$$

It can be seen that as $\varepsilon=1$, that is, in the absence of a porous medium, the governing equations, given in (1a)-(1c), reduce to the standard equations for free flows. In addition, some nondimensional parameters based on the governing equations can also be defined. The Richardson number is the dimensionless number that expresses the ratio of the buoyancy term, and we can define it in terms of the Grashof and Reynolds numbers as

$$
\mathrm{Ri}=\frac{\mathrm{Gr}}{\mathrm{Re}_{e}^{2}} .
$$

The Prandtl number can be defined as

$$
\operatorname{Pr}=\frac{J_{e}(\tau-0.5)}{\sigma\left(\tau^{1}-0.5\right)}
$$

where $\tau$ and $\tau^{1}$ are the relaxation times and the viscosity ratio $J_{e}$ and the ratio $\sigma$ between the heat capacities of the solid and fluid phases are set to 1 .

\section{Thermal Lattice Boltzmann Model for Porous Flows}

The evolution of the single-particle density distribution $f_{i}(x, t)$ of the velocity field and the temperature distribution $g_{i}(x, t)$ of the temperature field can be defined in the LBM as $[5,18]$

$$
\begin{aligned}
& f_{i}\left(x+e_{i} \delta t, t+\delta t\right)-f_{i}(x, t) \\
&=-\frac{1}{\tau}\left[f_{i}(x, t)-f_{i}^{\mathrm{eq}}(x, t)\right]+\delta t F_{i}, \\
& g_{i}\left(x+e_{i} \delta t, t+\delta t\right)-g_{i}(x, t) \\
&=-\frac{1}{\tau^{\prime}}\left[g_{i}(x, t)-g_{i}^{\mathrm{eq}}(x, t)\right],
\end{aligned}
$$

where $\delta$ is the time increment. Here, $f_{i}^{\mathrm{eq}}(x, t)$ and $g_{i}^{\mathrm{eq}}(x, t)$ are the equilibrium distribution functions (EDF) of $f_{i}(x, t)$ and $g_{i}(x, t)$, respectively, and are given by

$$
\begin{aligned}
& f_{i}^{\mathrm{eq}}(x, t)=\omega_{i} \rho\left[1+\frac{e_{i} \cdot u}{c_{s}^{2}}+\frac{e \cdot u^{2}}{2_{s}^{4}}-\frac{|u|^{2}}{2_{s}^{2}}\right], \\
& g_{i}^{\mathrm{eq}}(x, t)=\omega_{i} T\left[\sigma+\frac{e \cdot u}{c_{s}^{2}}\right],
\end{aligned}
$$

where $\omega_{i}$ is the weight coefficient and $c_{s}$ is the speed of sound. In the present work, we adopt the D2Q9 model, and the discrete velocities $e_{i}$ are given by

$e_{i}$

$$
= \begin{cases}0, & \text { for } i=0 ; \\ \left(\cos \frac{(i-1) \pi}{2}, \sin \frac{(i-1) \pi}{2}\right), & \text { for } i=1: 4 \\ \sqrt{2}\left(\cos \left[\frac{(i-5) \pi}{2}+\frac{\pi}{4}\right], \sin \left[\frac{(i-5) \pi}{2}+\frac{\pi}{4}\right]\right), & \text { for } i=5: 8 .\end{cases}
$$

Here, $c=\delta x / \delta t$ is the lattice spacing, the weight coefficients are $\omega_{0}=4 / 9, \omega_{i}=1 / 9(i=1-4)$, and $\omega_{i}=1 / 36$ ( $i=5-8)$, and the speed of sound is defined as $c_{s}=c / 3$. 

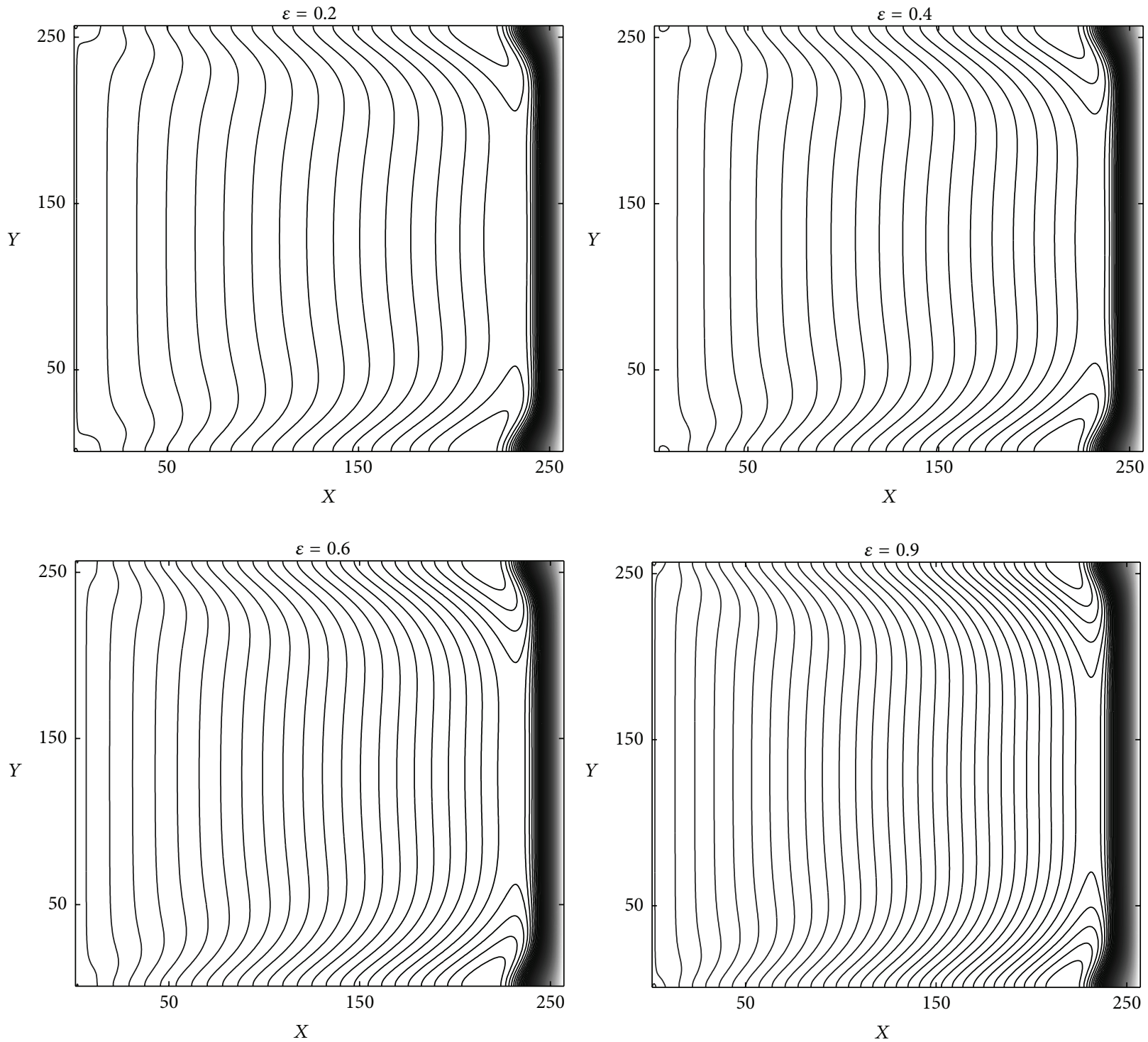

FIGURE 2: The isothermals for porosity: $\varepsilon=0.2,0.4,0.6,0.9$.

The forcing term $F_{i}$ in (6) is expressed as [5]

$$
\begin{aligned}
F_{i}= & \omega_{i} \rho\left(1-\frac{1}{2 \tau}\right) \\
& \cdot\left[\left(e_{i}-\frac{u}{\varepsilon}\right) \cdot \frac{F}{c_{s}^{2}}+\frac{\left(u \cdot e_{i}\right) \times\left(F \cdot e_{i}\right)}{\varepsilon c_{s}^{4}}\right],
\end{aligned}
$$

which gives the total force due to the presence of the porous medium and the other external force fields. Thus, the fluid density and velocity can be expressed as

$$
\begin{aligned}
& \rho=\sum f_{i}, \\
& u=\frac{v}{b_{0}+\sqrt{b_{0}^{2}+b_{1}|v|}},
\end{aligned}
$$

where $b_{0}, b_{1}$, and $v$ are given by

$$
\begin{aligned}
b_{0} & =\frac{1}{2}\left(1+\frac{\delta t}{2} \frac{\varepsilon}{\mathrm{DaRe}}\right), \\
b_{1} & =\frac{1}{2} \frac{\varepsilon F_{\varepsilon}}{\mathrm{Da}} \\
v & =\sum f_{i} \frac{e_{i}}{\rho}+\frac{\delta t}{2} \frac{\mathrm{Gr}}{\mathrm{Re}_{e}} k T .
\end{aligned}
$$

By applying the Chapman-Enskog expansion [5], the macroscopic equations, (6) and (7), become equivalent to those in [5]. For the problem discussed here, the velocity at the inlet is uniform; that is, $u=0.1$, and the velocity on the (adiabatic) horizontal sidewalls is equal to zero. 

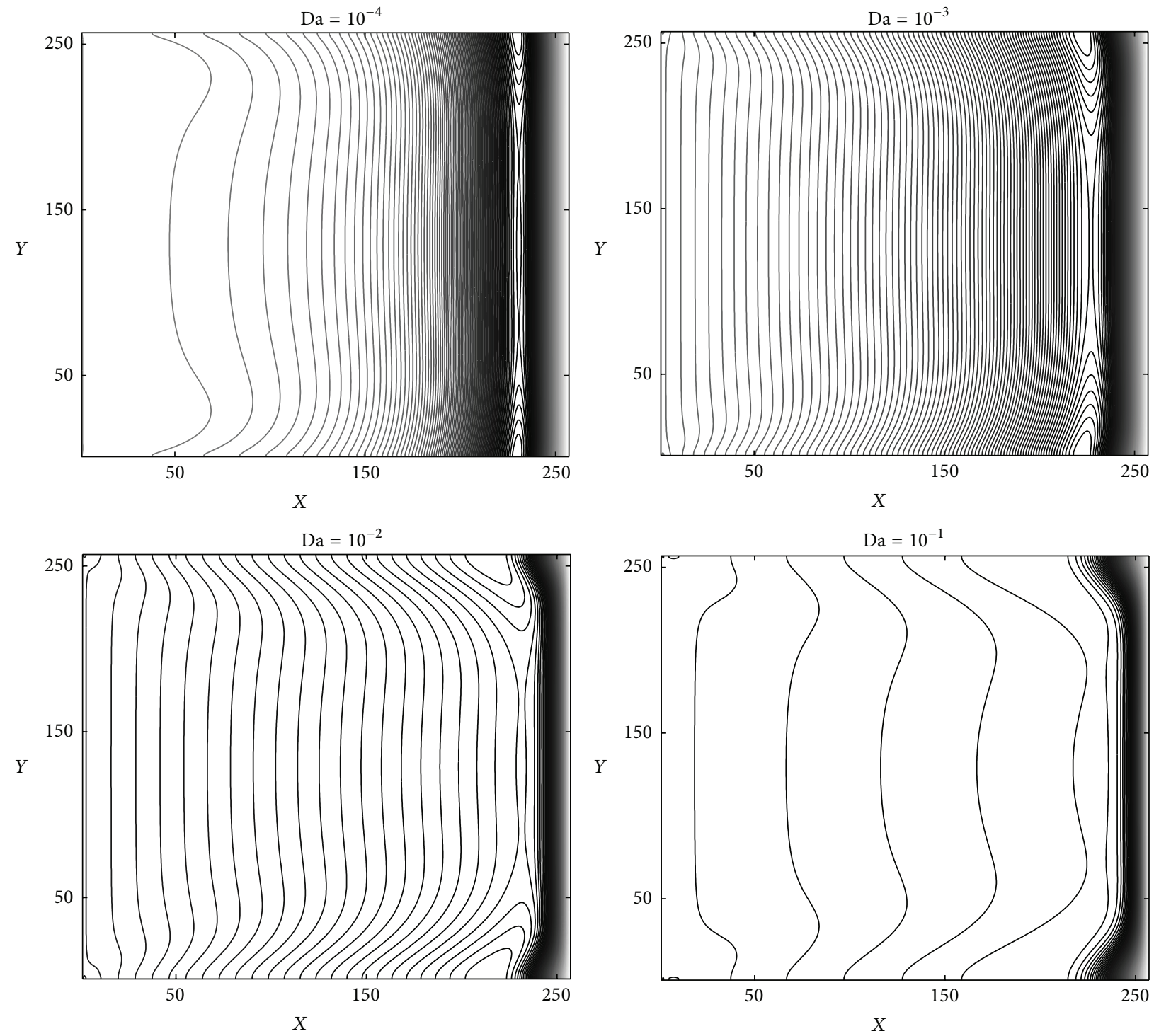

FIGURE 3: Isothermals for the Darcy numbers: $\mathrm{Da}=10^{-4}, 10^{-3}, 10^{-2}$, and $10^{-1}$.

\section{Numerical Results and Discussion}

To evaluate the accuracy of the present numerical method, we assume the following parameter values: $(\varepsilon=0.999)$; (Da $\left.=10^{6}\right) ; \operatorname{Pr}=0.71 ; \tau=0.833 ; \sigma=1 ; N_{x} \times N_{y}=257 \times 257 ; \operatorname{Re}=$ 100, 400, 1000; and $\mathrm{Ri}=1.0 \times 10^{-2}, 6.25 \times 10^{-4}$, and $1.0 \times 10^{-4}$.

The average Nusselt numbers at the hot wall for the present LBM are in good agreement with those reported in previous studies. A comparison is given in Table 1.

To investigate the effect of the porosity on the isothermals and average Nusselt numbers, calculations were carried out under conditions of $\mathrm{Ri}=0.0001, \mathrm{Da}=0.01$, and $\mathrm{Re}=100$ by varying the porosity $\varepsilon$ from 0.2 to 0.9 . The average Nusselt number at the isothermal walls is defined as [7]

$$
\overline{\mathrm{Nu}}=\int_{0}^{1} \frac{\partial T(x, 0)}{\partial y} d x
$$

TABLE 1: Comparison of the average Nusselt number at the hot wall in this work and that reported in previous studies for $\mathrm{Da}=10^{6}, \mathrm{Ri}=$ $10^{-2}, \varepsilon=0.999$, and $\tau=0.833$.

\begin{tabular}{lcccc}
\hline $\operatorname{Re}$ & Present & Reference [1] & Reference [6] & Reference [7] \\
\hline 100 & 2.04 & 1.97 & 1.94 & 2.01 \\
\hline 400 & 4.05 & 4.03 & 3.84 & 3.91 \\
\hline 1000 & 6.56 & 6.56 & 6.33 & 6.33 \\
\hline
\end{tabular}

This number is an important dimensionless parameter for describing the convective heat transport. Figure 2 shows that varying $\varepsilon$ has little impact on the temperature field. There is a steep temperature gradient in the horizontal direction at the right wall due to the driven convection and a weak uniform temperature gradient in the left-hand side of the cavity. As shown in Table 2, the average Nusselt numbers at the cold wall increase slightly with increasing porosity but those of the 

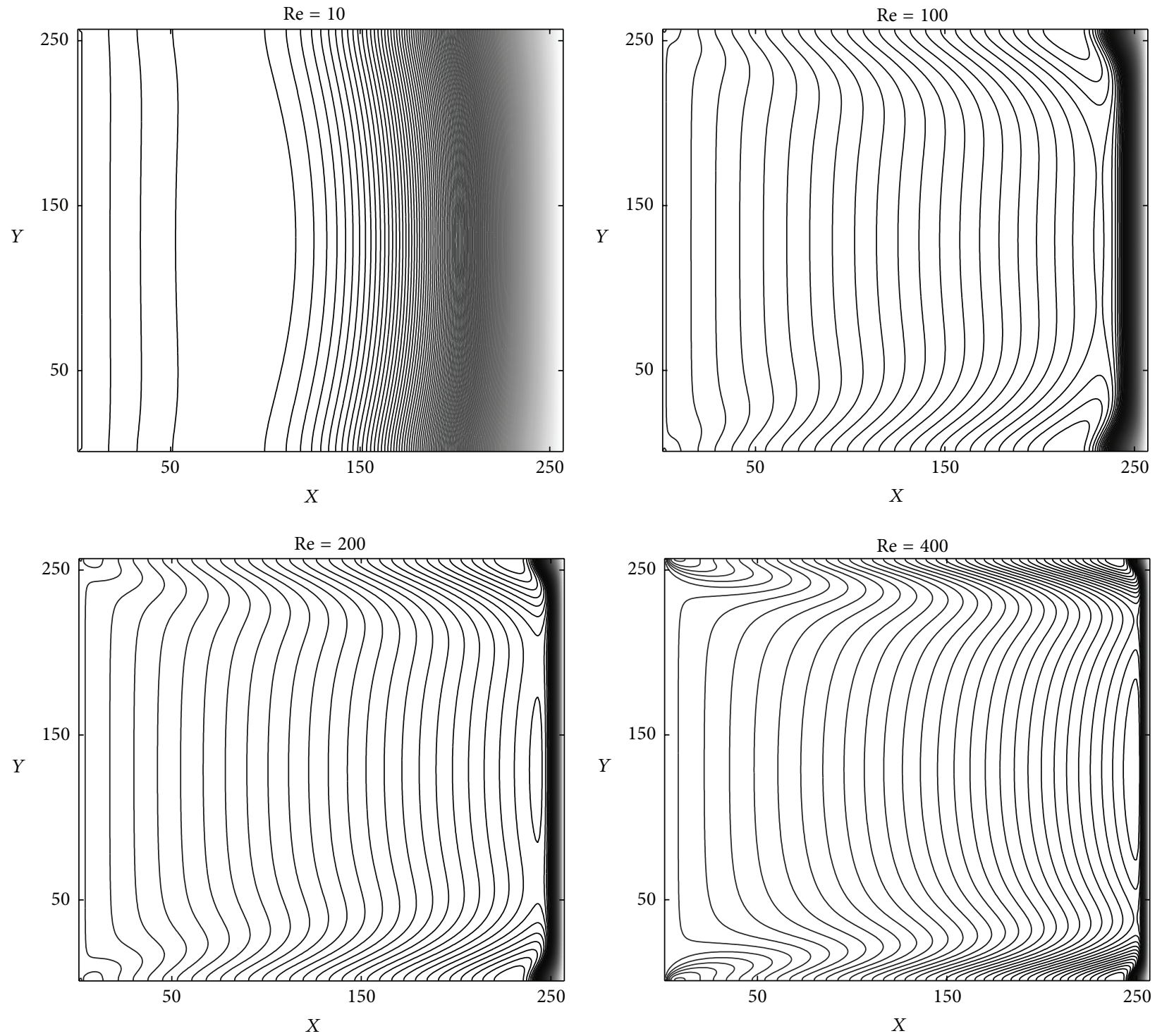

FIgURE 4: Isothermals for the Reynolds numbers: $\mathrm{Re}=10,100,200$, and 400.

TABle 2: Average Nusselt numbers at the hot and cold walls for different porosities.

\begin{tabular}{lcccc}
\hline$\varepsilon$ & 0.2 & 0.4 & 0.6 & 0.9 \\
\hline Cold wall & 0.091 & 0.111 & 0.134 & 0.167 \\
\hline Hot wall & 57.673 & 56.980 & 56.052 & 54.662 \\
\hline
\end{tabular}

hot wall decrease, which is consistent with the behavior of the temperature field.

The effect of the Darcy number on the temperature field is shown in Figure 3, where values of $\mathrm{Re}=100, \mathrm{Ri}=10^{-4}$, and $\varepsilon=$ 0.5 were assumed, and we see that the density of the boundary layer near the hot wall becomes thinner as $\mathrm{Da}$ increases. The data in Table 3 reveal that the average Nusselt numbers at the hot wall increase and those on the cold wall decrease as Da increases from $10^{-4}$ to $10^{-1}$. These results indicate that driven convection across the cavity is the dominant mechanism and that conduction heat transfer is strong at high $\mathrm{Da}$ values.

A similar phenomenon is also observed as the Reynolds number increases $\left(\mathrm{Da}=10^{-2}, \mathrm{Ri}=10^{-4}\right.$, and $\left.\varepsilon=0.5\right)$, as shown in Figure 4. As Re increases, the density of the isothermals near the hot wall reduces, and bending is observed due to the convection effect near the horizontal sidewalls. As can be seen in Table 4, the average Nusselt numbers near the hot wall increase rapidly with an increasing Re value, while there is only a small increase in the numbers near the cold wall.

The Richardson number represents the importance of natural convection relative to the forced convection, and its effect on the temperature field is shown in Figure 5. We see that there is little change in the field as Ri increases, but a strong temperature gradient appears suddenly in the upper right-hand region of the cell when $\mathrm{Ri} \leq 0.1$. This is consistent with the average Nusselt numbers listed in Table 5. 

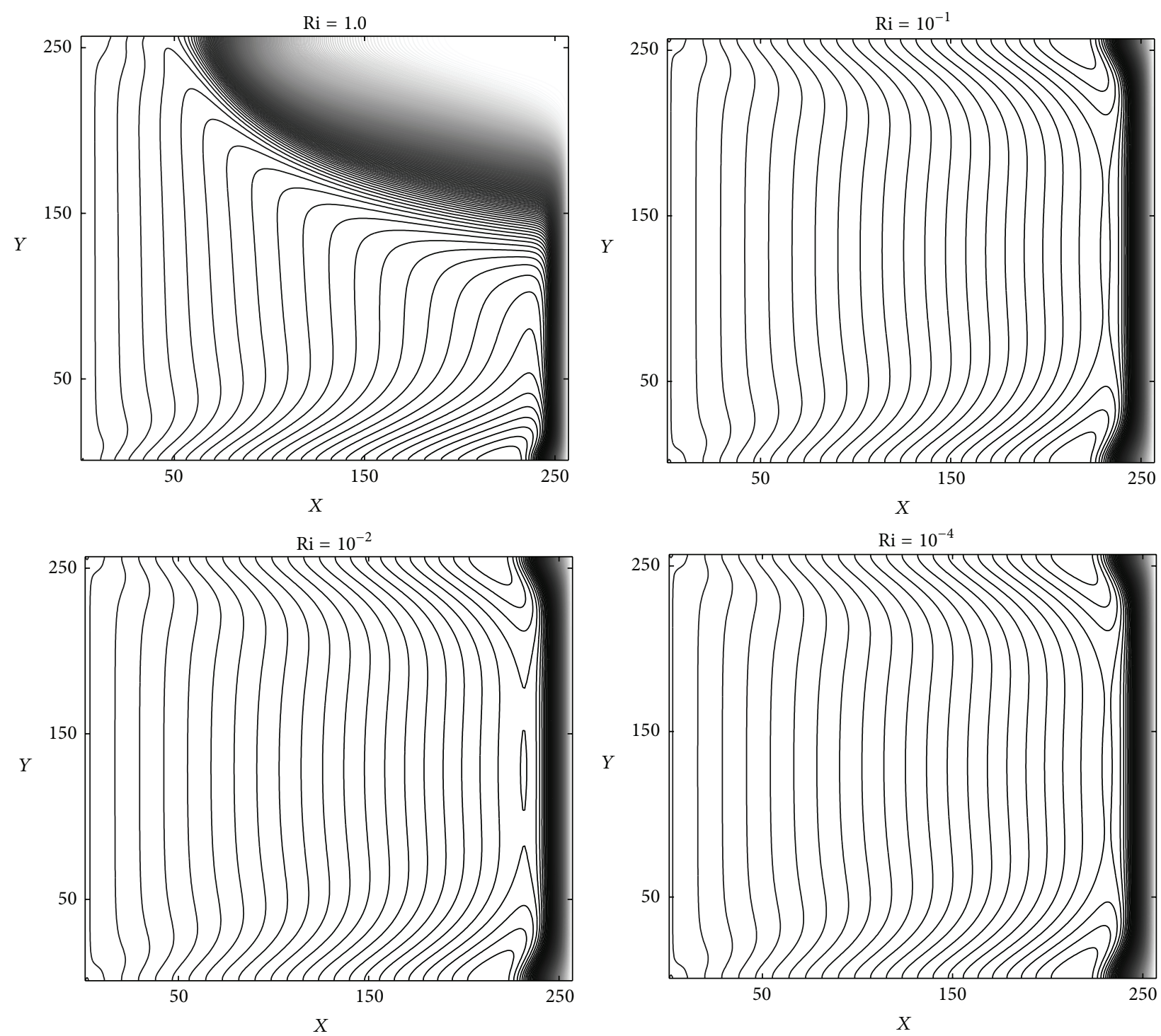

FIGURE 5: Isothermals for the Richardson numbers: $\mathrm{Ri}=10^{-4}, 10^{-2}, 10^{-1}$, and 1.0.

TABle 3: Average Nusselt numbers at the hot and cold wall for different Darcy numbers.

\begin{tabular}{lcccc}
\hline $\mathrm{Da}$ & $10^{-4}$ & $10^{-3}$ & $10^{-2}$ & $10^{-1}$ \\
\hline Cold wall & 1.238 & 0.393 & 0.122 & 0.028 \\
\hline Hot wall & 36.120 & 44.833 & 56.524 & 60.358 \\
\hline
\end{tabular}

TABle 4: Average Nusselt numbers at the hot and cold wall for different Reynolds numbers.

\begin{tabular}{lcccc}
\hline Re & 10 & 100 & 200 & 400 \\
\hline Cold wall & 0.091 & 0.122 & 0.139 & 0.146 \\
\hline Hot wall & 6.254 & 56.524 & 101.764 & 171.784 \\
\hline
\end{tabular}

TABle 5: Average Nusselt numbers at the hot and cold wall for different Richardson numbers.

\begin{tabular}{lcccc}
\hline $\mathrm{Ri}$ & $10^{-4}$ & $10^{-2}$ & $10^{-1}$ & 1.0 \\
\hline Cold wall & 0.122 & 0.122 & 0.122 & 0.134 \\
\hline Hot wall & 56.524 & 56.524 & 56.524 & 53.188 \\
\hline
\end{tabular}

Some additional insight can be gained by examining the horizontal velocity in the vertical midplane of the cavity (Figure 6). We see that there are two peaks in the velocity near the top and bottom walls that decrease as $\mathrm{Da}, \varepsilon$, or Re decreases, as shown in Figures 6(a), 6(b), and 6(c), respectively. This decrease in the velocity peaks is slightly weaker in the case of $\varepsilon$ and Re. Figure 6(d) shows the significant effect of Ri on the velocity. 


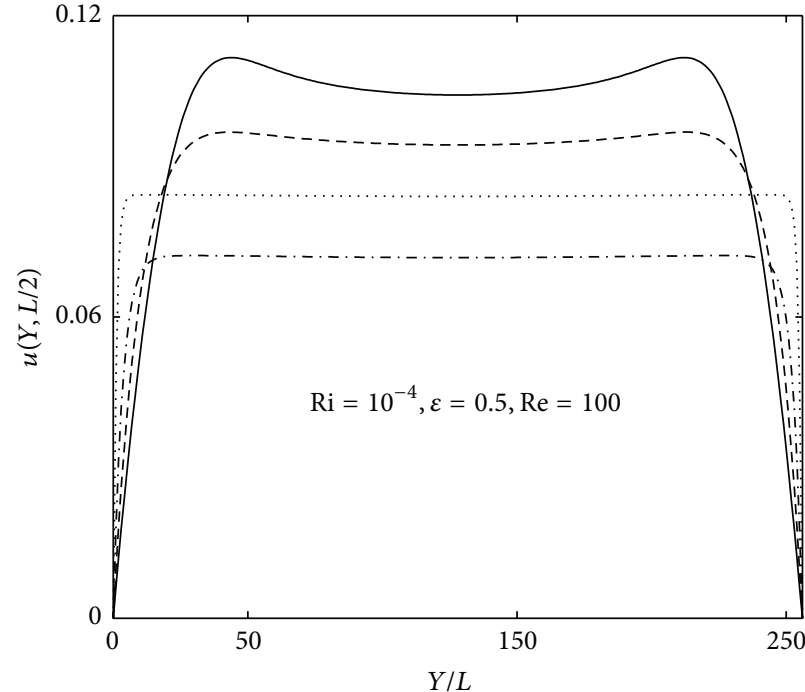

$$
\begin{array}{ll}
-\mathrm{Da}=10^{-1} & \cdots \mathrm{Da}=10^{-3} \\
--\mathrm{Da}=10^{-2} & \cdots \cdot \mathrm{Da}=10^{-4}
\end{array}
$$

(a)

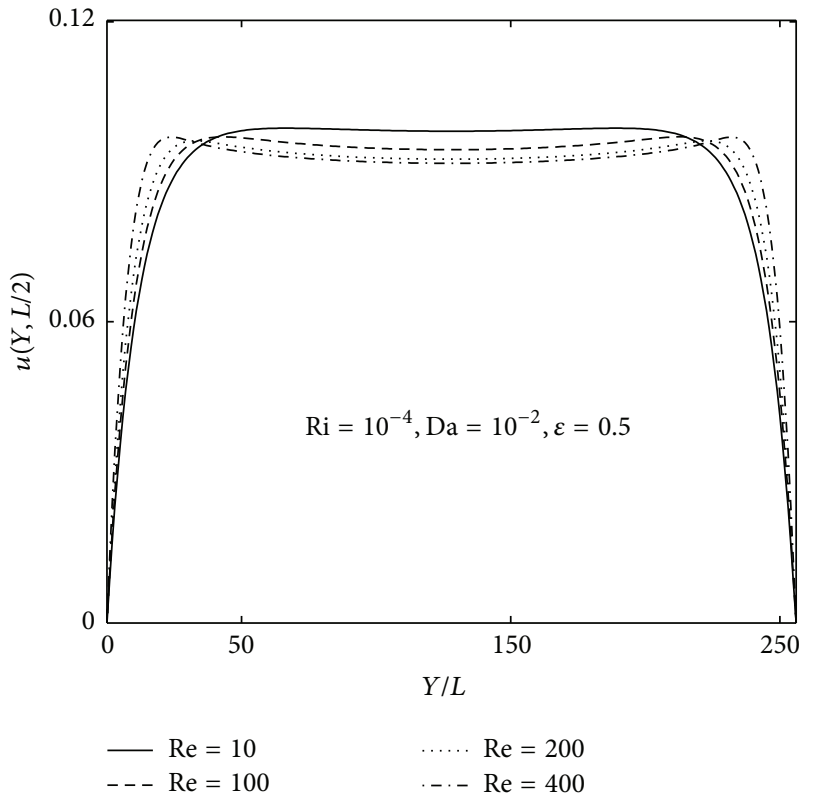

(c)

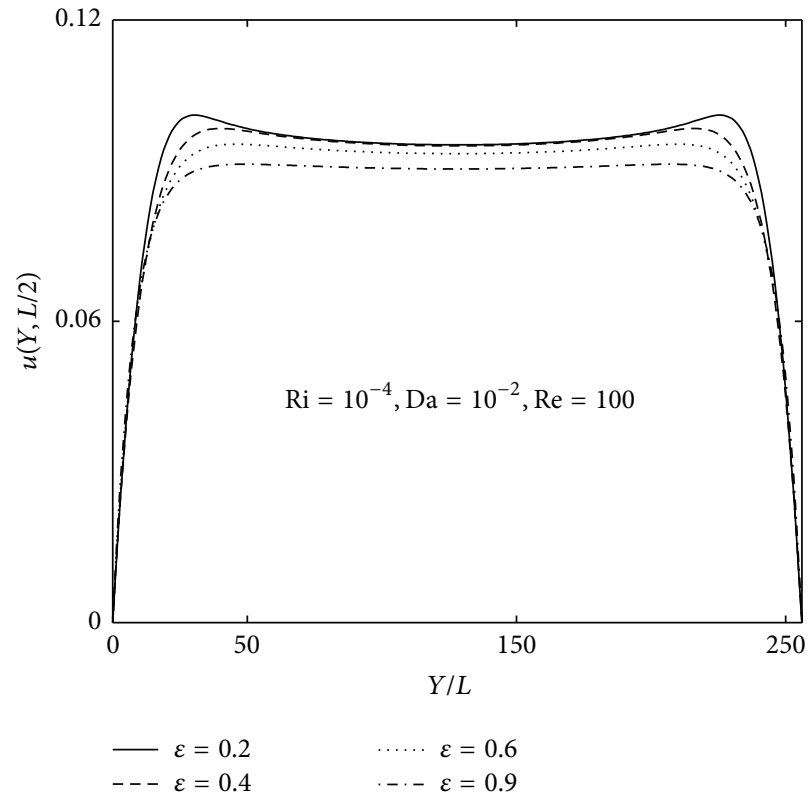

(b)

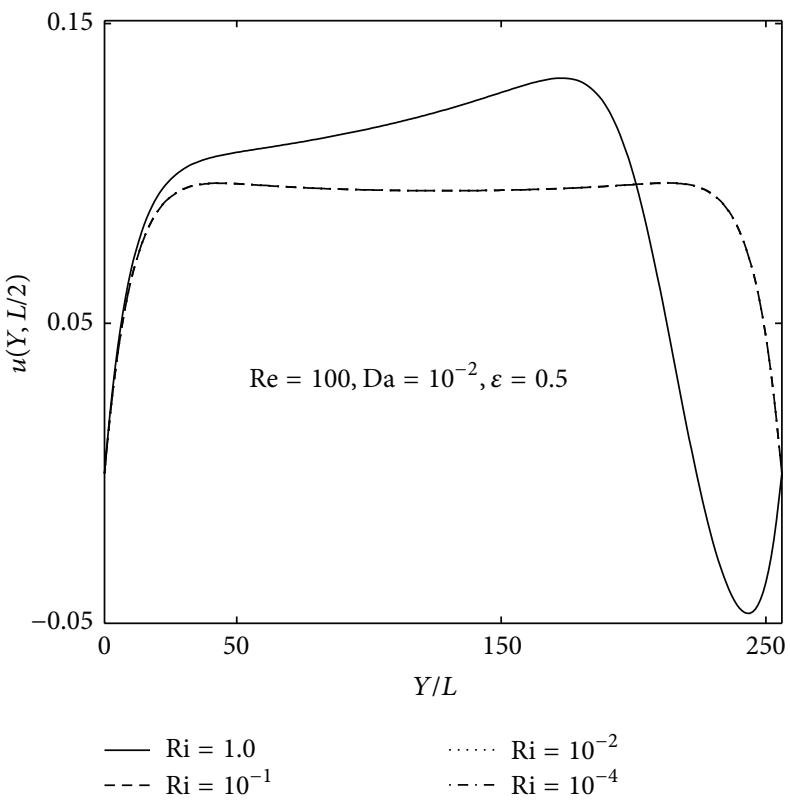

(d)

FIGURE 6: Horizontal velocity profiles in the vertical midplane for different (a) Darcy numbers, (b) porosities, (c) Reynolds numbers, and (d) Richardson numbers.

\section{Conclusions}

We have proposed an LBM for solving the driven convective heat transfer problem in porous media with a temperature field. The temperature distribution was obtained by solving an additional LBE, and the simulation results showed that variations in $\varepsilon$ and $\mathrm{Ri}$ had little impact on the temperature field and velocity profile, whereas significant changes were seen when $\mathrm{Ri}>0.1$. The influences of the Reynolds and Darcy numbers on the temperature field and the average Nusselt numbers near the hot wall were significant. Compared with traditional numerical methods, the LBM is more suitable for solving practical application problems in engineering.

\section{Conflict of Interests}

The authors declare that they have no financial or personal relationships with other people or organizations that can inappropriately influence their work; there is no professional or other personal interest of any nature or kind in any 
product, service, and/or company that could be construed as influencing the position presented in, or the review of, this paper.

\section{Acknowledgment}

This work was supported by the National Nature Science Foundation of China under Grants nos. U1262109, 51476145, and 51476146 which are gratefully acknowledged.

\section{References}

[1] Z. H. Chai, Z. L. Guo, and B. C. Shi, "Lattice Boltzmann simulation of mixed convection in a driven cavity packed with porous medium," in Computational Scienc-ICCS 2007, vol. 4448 of Lecture Notes in Computer Science, pp. 802-809, Springer, 2007.

[2] P. N. Shankar and M. D. Deshpande, "Fluid mechanics in the driven cavity," Annual Review of Fluid Mechanics, vol. 32, pp. 93-136, 2000.

[3] H. F. Oztop, "Combined convection heat transfer in a porous lid-driven enclosure due to heater with finite length," International Communications in Heat and Mass Transfer, vol. 33, no. 6, pp. 772-779, 2006.

[4] Z. Guo, B. Shi, and C. Zheng, "A coupled lattice BGK model for the Boussinesq equations," International Journal for Numerical Methods in Fluids, vol. 39, no. 4, pp. 325-342, 2002.

[5] Z. L. Guo and T. S. Zhao, "Lattice Boltzmann model for incompressible flows through porous media," Physical Review E, vol. 66, no. 3, Article ID 036304, 2002.

[6] K. M. Khanafer and A. J. Chamkha, "Mixed convection flow in a lid-driven enclosure filled with a fluid-saturated porous medium," International Journal of Heat and Mass Transfer, vol. 42, no. 13, pp. 2465-2481, 1999.

[7] J. Fan and H. W. K. Tsang, "Effect of clothing thermal properties on the thermal comfort sensation during active sports," Textile Research Journal, vol. 78, no. 2, pp. 111-118, 2008.

[8] L. Durlofsky and J. F. Brady, "Analysis of the Brinkman equation as a model for flow in porous media," Physics of Fluids, vol. 30, no. 11, p. 3329, 1987.

[9] V. Prasad and F. A. Kulacki, "Natural-convection in porousmedia bounded by short concentric vertical cylinder," ASME Journal of Heat Transfer, vol. 107, no. 1, pp. 147-154, 1985.

[10] R. Rajamani, C. Srinivas, P. Nithiarasu, and K. N. Seetharamu, "Convective heat transfer in axisymmetric porous bodies," International Journal of Numerical Methods for Heat and Fluid Flow, vol. 5, no. 9, pp. 829-837, 1995.

[11] P. Nithiarasu, K. N. Seetharamu, and T. Sundararajan, "Natural convective heat transfer in a fluid saturated variable porosity medium," International Journal of Heat and Mass Transfer, vol. 40, no. 16, pp. 3955-3967, 1997.

[12] P. Nithiarasu and K. Ravindran, "A new semi-implicit time stepping procedure for buoyancy driven flow in a fluid saturated porous medium," Computer Methods in Applied Mechanics and Engineering, vol. 165, no. 1-4, pp. 147-154, 1998.

[13] C. E. Hickox and D. K. Gartling, "A numerical study of natural convection in a vertical, annular, porous layer," International Journal of Heat and Mass Transfer, vol. 28, no. 3, pp. 720-723, 1985.

[14] N. Tatsuo, T. Toru, S. Mitsuhiro, K. Yuji, and O. Hiroyuki, "Numerical analysis of natural convection in a rectangular enclosure horizontally divided into fluid and porous regions," International Journal of Heat and Mass Transfer, vol. 29, no. 6, pp. 889-898, 1986.

[15] S. Chen and G. D. Doolen, "Lattice Boltzmann method for fluid flows," Annual Review of Fluid Mechanics, vol. 30, pp. 329-364, 1998.

[16] S. Succi, The Lattice Boltzmann Equation for Fluid Dynamics and Beyond, Numerical Mathematics and Scientific Computation, Oxford University Press, New York, NY, USA, 2001.

[17] S. Ergun, "Fluid flow through packed columns," Chemical Engineering Progress, vol. 48, pp. 89-94, 1952.

[18] Z. Guo and T. S. Zhao, "A lattice boltzmann model for convection heat transfer in porous media," Numerical Heat Transfer, Part B: Fundamentals, vol. 47, no. 2, pp. 157-177, 2005. 

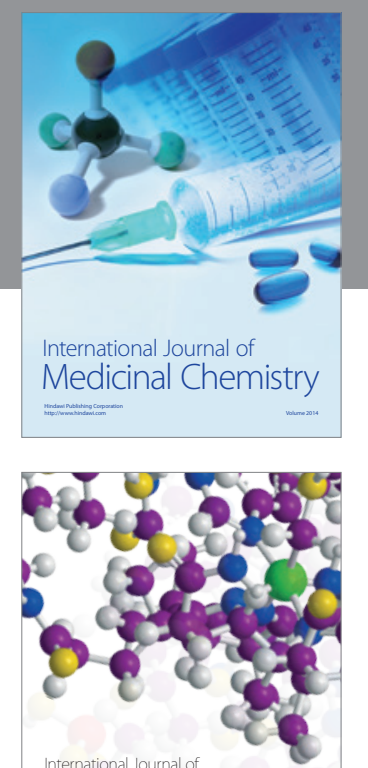

\section{Carbohydrate} Chemistry

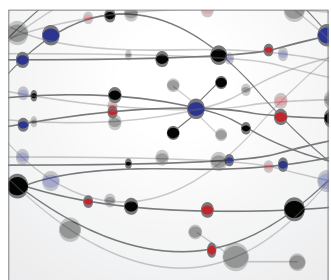

The Scientific World Journal
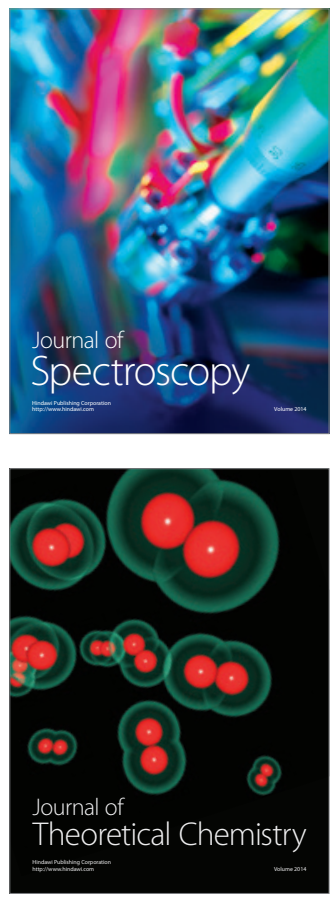
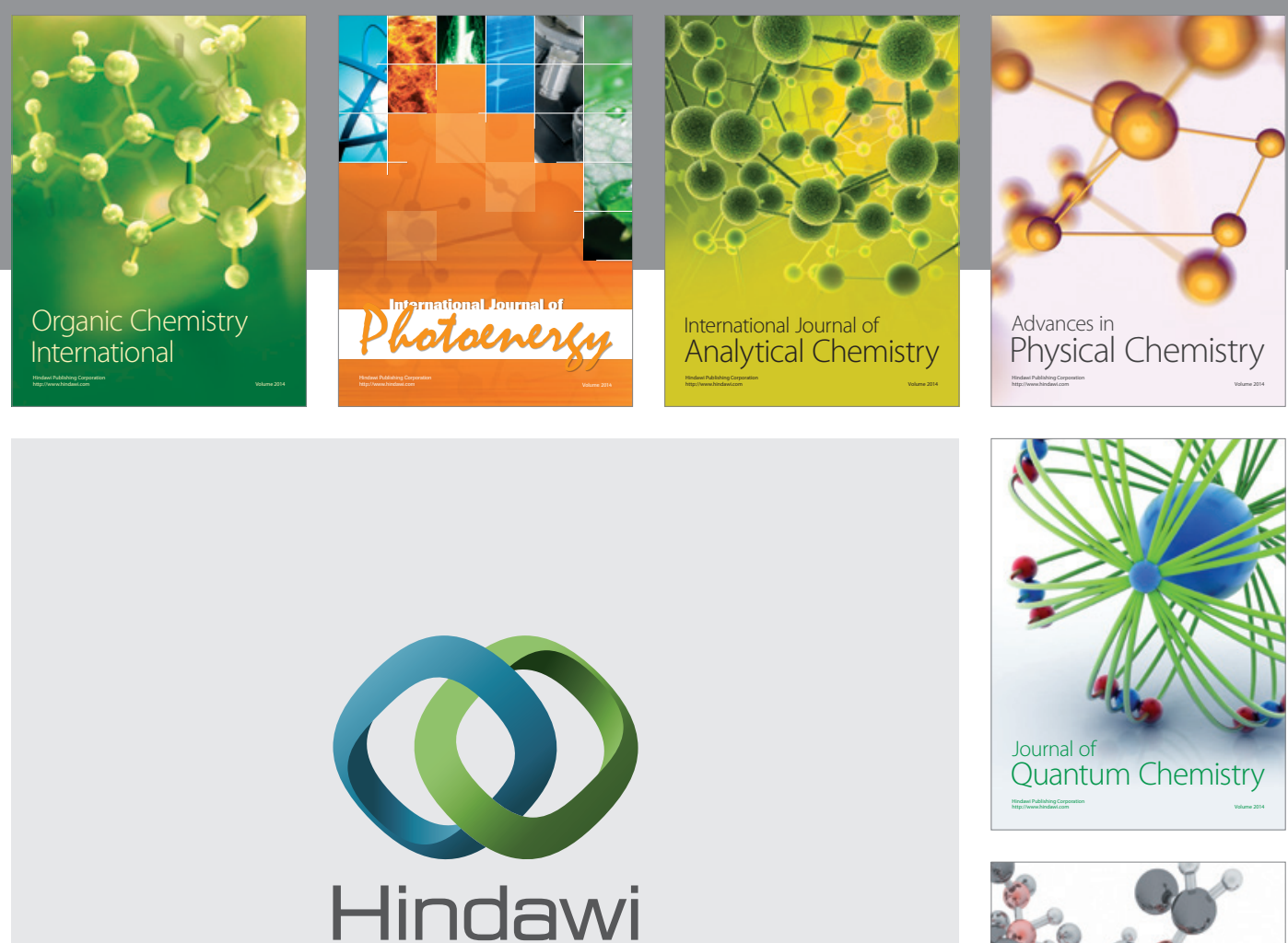

Submit your manuscripts at

http://www.hindawi.com

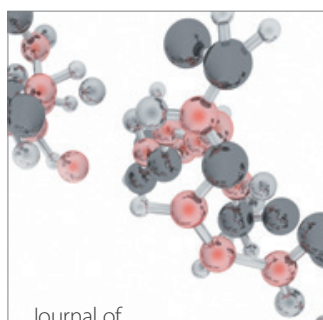

Analytical Methods

in Chemistry

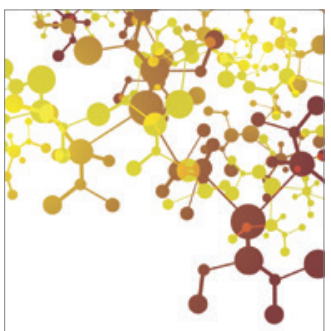

Journal of

Applied Chemistry

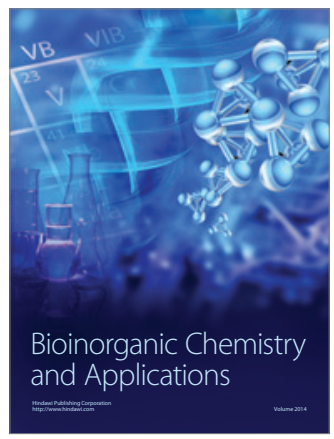

Inorganic Chemistry
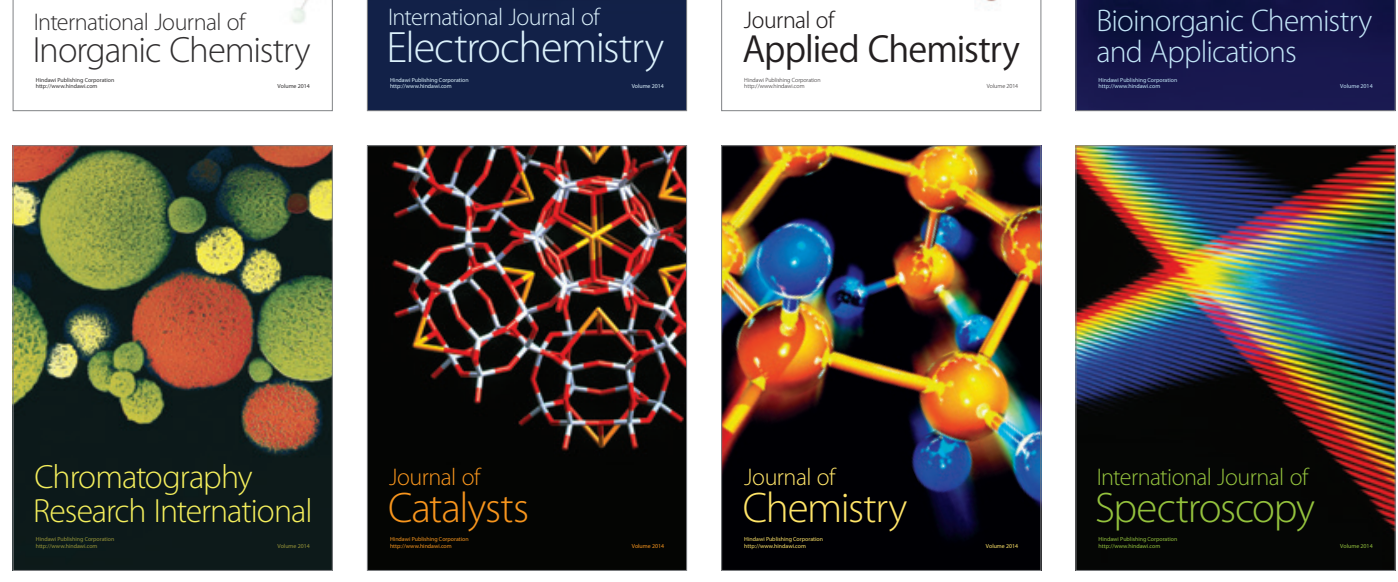